\title{
Shock Excitation of Emission Lines in GPS, CSS, and CSO Sources
}

Geoffrey V. Bicknell, ${ }^{1}$ Michael A. Dopita, ${ }^{2}$ and Christopher P. O'Dea ${ }^{3}$

${ }^{1}$ Astrophysical Theory Centre, Australian National University,

Canberra, ACT 0200, Australia

${ }^{2}$ Mt. Stromlo and Siding Spring Observatories, Weston PO, ACT 2611. Australia

${ }^{3}$ Space Telescope Science Institute, 3700 San Martin Drive, Baltimore, MD 21218, USA

\section{Introduction}

The classes of radio-loud AGN known as Gigahertz-peak spectrum (GPS), compact steep spectrum (CSS), and compact symmetric objects (CSO) represent an important sub-class of AGN, constituting, for example, $24 \%$ of the Baker, Hunstead, \& Brinkmann (1995) sample. Table 1 summarizes some of their key properties.

Table 1. Properties of GPS, CSS, and CSO Sources

\begin{tabular}{|c|c|c|}
\hline Source & Characteristics & General Properties \\
\hline GPS & $\begin{array}{l}\text { Peak in radio spectrum } \\
\text { at } \mathrm{GHz} \text { frequencies } \\
<\text { Size }>\approx 350 \mathrm{pc} \\
<\text { Pressure }>\approx 10^{-6} \mathrm{dyn} \mathrm{cm}^{-2}\end{array}$ & $\begin{array}{l}\left\langle P_{5 \mathrm{GHz}}>\approx 10^{27.5} \mathrm{~W} \mathrm{~Hz}^{-1}\right. \\
\mathrm{RM} \approx 0-1000 \mathrm{rad} \mathrm{m}^{-2}\end{array}$ \\
\hline CSS & $\begin{array}{l}\text { Steep spectrum at } \mathrm{GHz} \text { frequencies } \\
\text { Spectral peak at } \sim 100 \mathrm{MHz} \\
<\text { Size }>\approx 10 \mathrm{kpc} \\
<\text { Pressure }>\approx 10^{-8} \mathrm{dyn} \mathrm{cm}^{-2}\end{array}$ & $\begin{array}{l}\text { Strong forbidden line } \\
\text { spectrum } \\
\text { Forbidden-line widths } \\
\sim 500-1000 \mathrm{~km} \mathrm{~s}^{-1}\end{array}$ \\
\hline CSO & $\begin{array}{l}\text { Compact, symmetric } \\
\text { FR II-like morphology }\end{array}$ & \\
\hline
\end{tabular}

It is now clear (e.g., Stanghellini et al. 1996) that GPS and CSS sources differ only in the location of the peak frequency. Moreover, every CSO is a GPS source, and while the reverse is not strictly true, there is certainly a large overlap between GPS sources and CSOs and we assume that the interaction of the respective jets with the ISM in these classes is similar. For some time, it has been debated whether such sources are young or confined. Begelman (1996) proposed an elegant solution to this problem by constructing a model involving the interaction of young radio lobes with a dense power-law atmosphere. In Begelman's view, the sources are relatively young ( $\left.\sim 10^{6} \mathrm{yrs}\right)$ compared to FR II radio 
galaxies, and his model provides a satisfactory explanation for the luminositysize relation. We have developed this model for the purpose of explaining the correlation between emission-line luminosity and radio power (Gelderman \& Whittle 1996) and peak turnover frequency and size (Stanghellini et al. 1996), thereby unifying the radio and optical properties of these objects.

\section{Begelman's Dynamical Model for Compact Symmetric Objects}

Begelman's (1996) model for 'baby Cygnus As' (modified to allow for adiabatic losses in the expanding cocoon) provides the basis for our modeling of the emission-line luminosity and peak-frequency-size relation. The averaged pressure at the head of the lobe is assumed to be a constant factor $\zeta$ times the average pressure in the rest of the lobe. Taking the ambient density, $\rho_{\mathrm{a}}=\rho_{0}\left(x / x_{0}\right)^{-\delta}$, where $\rho_{0}$ and $x_{0}$ are fiducial values and the jet energy flux to be $F_{E}$, this model implies for the distance $x_{\mathrm{h}}(t)$ of the hotspot from the core and for the average cocoon pressure $P_{c}(t)$

$$
x_{\mathbf{h}}=x_{0} \xi^{1 /(5-\delta)} \quad \text { and } \quad P_{\mathrm{c}}=P_{0} \xi^{(2-\delta) /(5-\delta)}
$$

where

$$
\xi=\frac{(5-\delta)^{3} \zeta^{2}}{18 \pi(8-\delta)}\left(\frac{F_{E} t^{3}}{\rho_{0} x_{0}^{5}}\right) \quad \text { and } \quad P_{0}(t)=\frac{9}{\zeta(5-\delta)^{2}} \rho_{0}\left(\frac{x_{0}}{t}\right)^{2}
$$

For the above-quoted average size and pressure for a GPS source, the jet energy flux and ambient density are $\sim 5 \times 10^{44} t_{6}^{-1} \mathrm{ergs} \mathrm{s}^{-1}$ and $100 t_{6}^{2} \mathrm{~cm}^{-3}$, respectively. Energy fluxes $\sim 10^{45} \mathrm{ergs}^{-1}$ are consistent with an age $\sim 5 \times 10^{5} \mathrm{yr}$ and a ratio $\kappa_{5}$ of monochromatic power at $5 \mathrm{GHz}$ to jet energy flux $\sim 10^{-11}-10^{-10.5}$, a factor of a few higher than is normally assumed for FR II sources, but consistent with the luminosity-size evolution suggested by Begelman (1996).

The lateral expansion velocity of the cocoon as a function of distance is given by

$$
V_{\mathrm{c}}=V_{0}\left(\frac{x_{\mathrm{h}}}{x_{0}}\right)^{(\delta-2) / 3}
$$

where

$$
V_{0} \approx 1500\left(\frac{6}{8-\delta}\right)^{1 / 3} \zeta^{1 / 6}\left[\frac{F_{E, 45}}{n_{\mathrm{H}, 0}}\right]^{1 / 3} \mathrm{~km} \mathrm{~s}^{-1},
$$

$10^{45} F_{\mathrm{E}, 45}$ ergs s$^{-1}$ is the energy flux, and $n_{\mathrm{H}, 0}$ is the Hydrogen number density at $x_{0}=1 \mathrm{kpc}$. For $F_{E, 45} / n_{\mathrm{H}, 0} \approx 0.1$, the expansion velocity is of order the line widths observed by Gelderman \& Whittle (1996).

\section{Emission from Radiative Shocks}

The bow shock advancing into the ISM is radiative for ambient densities greater than $\sim 10 \mathrm{~cm}^{-3}$ and ages $\lesssim 10^{6-7}$ yrs. The total continuum plus emissionline luminosity $L_{T}$ and [O III] luminosity $L\left(\left[\mathrm{O}_{\mathrm{III}}\right]\right)$ from the shocked gas and 

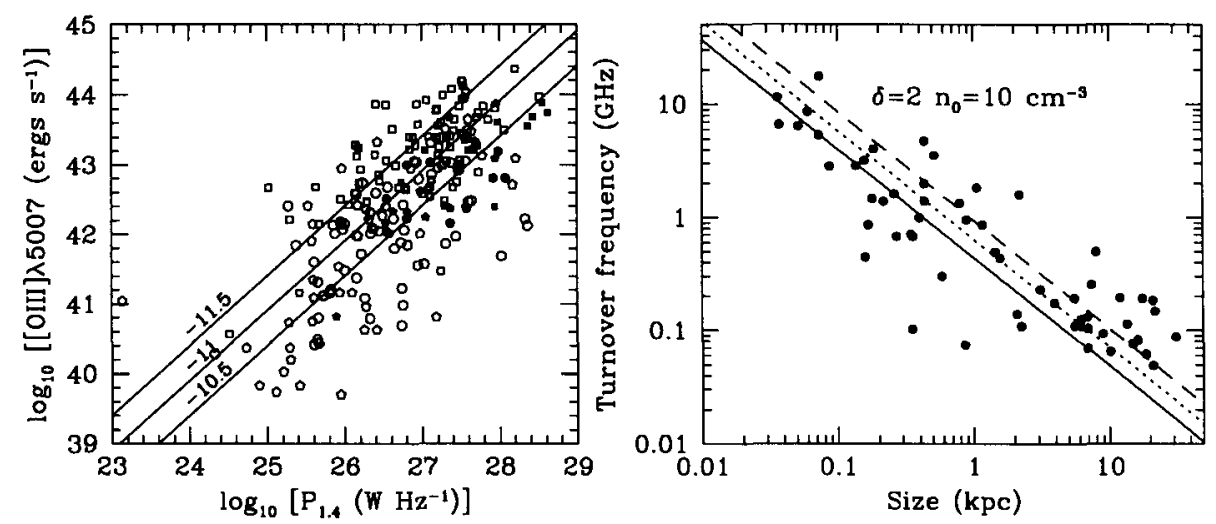

Figure 1. Left: Predicted [O III]-luminosity/radio-power relation for various values of $\log \kappa_{1.4}$ compared to the data for radio-loud AGN. Filled circles, filled squares, open circles and open squares represent CSS radio galaxies, CSS quasars, radio galaxies and radio-loud quasars, respectively, from Gelderman \& Whittle (1996); filled hexagons and open hexagons are unresolved sources and radio galaxies from Tadhunter et al. (1993) and Morganti et al. (1993). Right: Turnoverfrequency versus size relation compared to data from Stanghellini et al. (1996). Jet energy fluxes of $10^{45}, 10^{45.5}$ and $10^{46} \mathrm{ergs} \mathrm{s}^{-1}$ are represented by solid, dotted, and dashed lines, respectively.

photoionized precursor were calculated for a range of shock velocities using the MAPPINGS II emission-line code (Sutherland \& Dopita 1993) and are given by (in units of ergs $\mathrm{s}^{-1}$ )

$$
L_{T}=1.1 n_{\mathrm{H}} V_{3}^{3} A_{\mathrm{sh}} \quad L([\mathrm{O} \mathrm{III}])=2.3 \times 10^{-2} n_{\mathrm{H}} V_{3}^{3} A_{\mathrm{sh}},
$$

where $A_{\mathrm{sh}} \mathrm{cm}^{2}$ is the shock area. Equating $L_{T}$ to the rate of work, $3 /(8-\delta) F_{E}$ done by the expanding lobe gives

$$
L([\mathrm{O} \mathrm{III}])=8.2 \times 10^{42}\left(\frac{6}{8-\delta}\right)\left(\frac{\kappa_{1.4}}{10^{-11}}\right)^{-1}\left(\frac{P_{1.4}}{10^{27} \mathrm{~W} \mathrm{~Hz}^{-1}}\right) \text { ergs s}^{-1}
$$

where $\kappa_{1.4}$ is the ratio of $1.4 \mathrm{GHz}$ radio power to jet energy flux. A comparison of this relation with the [O III] luminosities for radio galaxies and quasars from Gelderman \& Whittle (1996) and radio galaxies from Tadhunter et al. (1993) and Morganti, Killeen, \& Tadhunter (1993), is shown in the left-hand panel of Fig. 1. Established and candidate GPS, CSS, and CSO sources are indicated by filled symbols. Good agreement is obtained for $\kappa_{1.4} \approx 10^{-10.5}$. However, the [O III] fluxes of GPS sources are substantially reddened (Baker \& Hunstead 1996) so that correspondingly smaller values of $\kappa_{1.4}$ may be relevant. Similar good agreement is obtained for the $\mathrm{H} \alpha+[\mathrm{N} \mathrm{II}]$ data from Gelderman \& Whittle (1996); see Bicknell, Dopita, \& O'Dea (1996). 


\section{The Radio Spectra and the Peak-Frequency-Size Relation}

Both the anticorrelation between peak frequency and size and the power-law slope at low frequency can be attributed to free-free absorption by the ionized shock and precursor regions. The free-free optical depth $\tau_{\nu}=a \nu_{9}^{-2.1}$, where $a=1.1 \times 10^{-25} \int n_{e}^{2} T_{4}^{-1.35} \mathrm{dl}$. The contributions to $a$ from uniform shock and precursor zones are $2.0 \times 10^{-3} V_{3}^{2.3} n_{\mathrm{H}}$ and $1.0 \times 10^{-3} V_{3}^{1.5} n_{\mathrm{H}}$ respectively, where $n_{\mathrm{H}} \mathrm{cm}^{-3}$ is the pre-shock Hydrogen density and $V_{3}$ is the shock velocity in units of a thousand $\mathrm{km} \mathrm{s}^{-1}$. A uniform medium surrounding the lobes would give an $\exp \left[-a \nu^{-2.1}\right]$ cutoff to the spectrum. However, if we assume that $a$ is distributed as a truncated power-law (probability density function $\propto a^{p}$ for constant $p$ and $\left.0<a<a_{0}\right)$, then the flux density is

$$
F_{\nu}=A\left(\frac{\nu}{\nu_{0}}\right)^{2.1(p+1)-\alpha} \gamma\left(p+1,\left(\nu / \nu_{0}\right)^{-2.1}\right),
$$

where $A$ is a constant, $\alpha$ is the high-frequency spectral index, $\nu_{0}^{2.1}=a_{0}$, and $\gamma(p+1, x)$ is the incomplete gamma function. This spectrum peaks at $\nu \approx \nu_{0}$ and the low-frequency spectral index is $\alpha-2.1(p+1)$. Evaluating $p$ from the lowfrequency spectral indices estimated by Stanghellini et al. (1996) gives $\langle p\rangle \approx$ -0.2 , close to a uniform distribution. It is most likely that any broad distribution of optical depths will lead to a spectrum of the required form. We take $\langle a\rangle$ to be the value predicted by the uniform one-dimensional MAPPINGS models so that the peak frequency is

$\nu_{p} \approx 1.1\left(\frac{p+2}{p+1}\right)^{0.48}\left[2.0 \times 10^{-3} V_{3}^{2.3}+1.0 \times 10^{-3} V_{3}^{1.5}\right]^{0.48} n_{0}^{0.48}\left(\frac{x}{\mathrm{kpc}}\right)^{-0.48 \delta}$.

This relationship is plotted (for $\delta=2$ ) and favorably compared to the Stanghellini et al. (1996) data in the right-hand panel of Fig. 1. It can also be shown that the magnetionic medium surrounding the radio lobes produces substantial Faraday depolarization explaining another important characteristic of these sources. Thus, using quite straightforward physics, we have shown that the radio and optical properties of this class of AGN can be unified.

\section{References}

Baker, J. C., \& Hunstead, R. W. 1996, ApJ, 452, L95.

Baker, J. C., Hunstead, R. W., \& Brinkmann, W. 1995, MNRAS, 277, 553.

Begelman, M.C. 1996, in Cygnus A: Study of a Radio Galaxy, ed. C. L. Carilli \& D.A. Harris (Cambridge University Press: Cambridge).

Bicknell, G. V., Dopita, M.A., \& O'Dea, C.P., ApJ, submitted.

Gelderman, R., \& Whittle, M. 1996, ApJ, in press.

Morganti, R., Killeen, N.E. B., \& Tadhunter, C. N. 1993, MNRAS, 263, 1023.

Stanghellini, C., O'Dea, C., Fanti, R., Baum S. A. 1996, in preparation.

Sutherland, R. S., and Dopita, M. A. 1993, ApJS, 88, 253.

Tadhunter, C. N., Morganti, R., di Serego Aligheri, S., Fosbury, R.A.E., \& Danziger, I. J. 1993, MNRAS, 263, 999. 